\title{
The effect of repetition and similarity on sequence learning
}

\author{
Padraic Monaghan \\ Lancaster University, Lancaster, England \\ AND \\ CHRIS RowSON \\ University of York, York, England
}

\begin{abstract}
Repetition is a pervasive feature of children's environments, and may be an important contributor to learning such complex sequential structures as language. Endress, Dehaene-Lambertz, and Mehler (2007) found that repeated tone sequences were learned more easily than sequences containing ordinal relations, but there have been no direct comparisons of repeating sequences versus sequences that contain similar, but not identical, stimuli. In Experiment 1, we compared learning from repeating tone sequences to learning from tones that varied in similarity, and confirmed that repetition is a special case for learning. In Experiment 2 we showed that the learning distinction between repeated and similar elements is not affected by whether similarity is variable. We conclude by indicating that repetition provides an important constraint on learning, and we discuss the extent to which such constraints are consistent with general-purpose statistical learning mechanisms.
\end{abstract}

Repetitions in stimuli have important consequences for perception and cognition (e.g., Henson, 1998; Kanwisher, 1987), and are thus likely to provide important constraints for learning. Repetition is a likely candidate as a useful feature for sequence learning, particularly for computing complex sequences such as those engaged for language learning (e.g., Saffran, 2003). Children's books and childdirected speech, for instance, are replete with instances of repeating words and phrases (Papousek, Papousek, \& Haekel, 1987), which makes such repetition seem unlikely to be an accidental property. Determining the precise role of repetition for learning sequence structure is therefore important in order to establish the identity of contributors to learning structure from sequences.

In a recent, intriguing study, Endress, DehaeneLambertz, and Mehler (2007) examined the influence of repetitions in learning sequences of tones. They compared participants' learning of patterns of three musical tones that varied in terms of whether they contained repetitions or "ordinal" relations (in this case, whether the third tone was higher or lower in pitch than the first tone). Participants listened to four sets of three tones, then judged whether a fifth set of tones had the same or a different structure. In the repetition condition, participants learned to distinguish low-high-low or high-lowhigh tones (ABA) from low-high-high or high-low-low (ABB) patterns; examples of these stimuli are shown in the top two rows of Figure 1. In the ordinal condition, the distinction was between low-high-medium (LHM) and medium-high-low (MHL) tones; see the third and fourth rows of Figure 1. Endress et al. found that participants performed more accurately in the repetition condition than in the ordinal condition. However, there are several ways in which the repeating and the ordinal sequences vary in Endress et al.'s study, not only in terms of whether they contained a repetition; we summarize these in the introduction to Experiment 1. Our first aim for the present experiments was to provide a more rigorous test of the advantage of learning from sequences containing repetition, by directly comparing repeating and nonrepeating sequences.

A second aim of the present study was to determine whether repetition of identical stimuli is a special case for learning, or whether it is better conceived as one endpoint of a continuum of similarity. Previous studies of statistical learning in language-like sequences have provided evidence for similarity (rather than identical repetition) assisting in sequence learning. Newport and Aslin (2004) tested participants' ability to learn nonadjacent dependencies between two syllables when the intervening syllable was kept constant. They found that learning was best when the first and third syllables shared phonological properties, and varied the extent to which they did so. In a related finding, Onnis, Monaghan, Richmond, and Chater (2005) found that the nonadjacency structure of words in continuous speech was detected only when the dependent syllables shared phonological properties. Bonatti, Peña, Nespor, and Mehler (2005) also found learning of sequence structure when the dependent elements of speech-like sequences carried similarity in either the consonants or vowels.

P. Monaghan, p.monaghan $@$ lancaster.ac.uk 


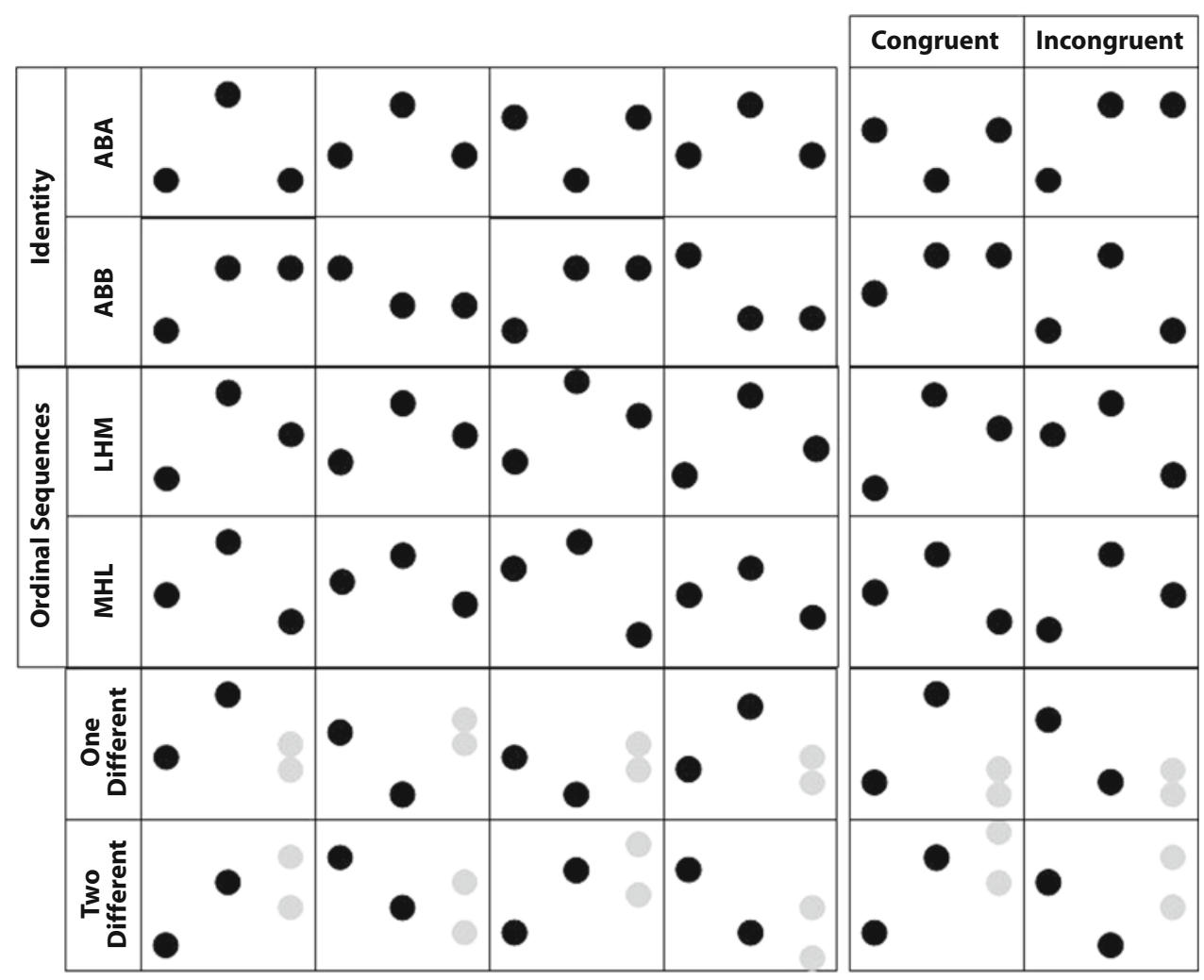

Figure 1. ABA and $A B B$ tone patterns for repetition condition and MHL and LHM patterns from Endress et al. (2007), and ABA pattern for one-different and ABB pattern for two-different conditions in Experiment 1. Black circles indicate the pitch of the tone, and gray circles indicate the two possible pitches for the third tone.

Other studies have directly tested repetition in sequence learning and have found advantages for certain patterns of repetition in both infants (Marcus, Vijayan, Rao, \& Vishton, 1999) and recurrent neural networks (Altmann, 2002). Yet there has been no study that directly compares the effects of similarity with repetition. One possibility is that increasing similarity corresponds to increasing accuracy of learning, so the effect of repetition is quantitatively continuous, with sequences containing similar stimuli. Another possibility is that repetition is instead qualitatively distinct from sequences, so the distinction in learning from sequences containing similar stimuli would be a step change from sequences containing precise repetitions. Establishing this aspect of repetition processing has implications for the constraints required in an adequate cognitive model of sequence learning. If repetition is continuous with similarity, this conforms to standard views of statistical learning mechanisms applied to sequences, where the similarity of representation of stimuli is related to their featural similarity; it is a view coherent with one of the foundational principles of connectionist models (Hopfield, 1982; Kohonen, 1988). However, if repetition is qualitatively distinct from similarity, this presents a challenge to models of statistical learning that represent stimuli along a continuum according to similarity.
A further aim of the present study was to tease apart the contribution of similarity between two items, and the contribution of variability in the predictability of the similar elements. Sequences that contain similar, but not identical, stimuli may be predictably different, as in the Newport and Aslin (2004) and Onnis et al. (2005) studies, in which the stimuli with similar properties are reliably co-occurrent. Alternatively, similar sequence stimuli may differ in their predictability - where the extent of the similarity is variable. Such differences in predictability may be an important contributor to potential distinctions between repeating and nonrepeating sequences.

Experiment 1 compares learning of sequences where either the first and third or second and third tones differ by zero, one, or two semitones, though the final tone can be higher or lower. This experiment examines the combined influence of similarity and variability of the solution space. Experiment 2 tests only the effect of similarity, where the third tone was either identical to or higher by one or two tones from the first or second tone.

\section{EXPERIMENT 1 \\ Learning From Similarity and Variability}

We used a paradigm related to Endress et al.'s (2007) study to determine the role of repetition in sequence learn- 
ing. However, the repeated versus ordinal sequence comparison in Endress et al.'s study introduced differences in addition to whether the sequence contained repetitions or not. First, $\mathrm{ABA}$ and $\mathrm{ABB}$ sequences could be differentiated just by attending to the final two tones in each sequence and responding whether they were the same or different. For ordinal sequences, both MHL and LHM sequences could only be contrasted by reference to the first and third tones (note that participants were trained on either the repetition or the ordinal sequences). Such nonadjacencies are more difficult to learn than adjacent dependencies (Gómez, 2002; Onnis et al., 2005; see also indirect evidence in Peña, Bonatti, Nespor, \& Mehler, 2002). In a related phenomenon, the nonadjacent dependencies in the ordinal sequences were likely to be difficult to identify because of the lack of variation in the second tone (always a tone of higher pitch). In the repeating sequences, the second tone may rise or fall with respect to the first tone. Gómez found that increasing variability in the intervening element in three-word sequences improved the learning of the relationship between the first and the third word. Lack of variability promotes bigram monitoring (Onnis, Christiansen, Chater, \& Gómez, 2003), an ineffective strategy for the ordinal sequences.

Second, the search space of the final tone was greater in the ordinal sequences than in the repetition sequences. In the ordinal condition, the final tone of each sequence can be anywhere below the first tone in MHL sequences, and anywhere between the first two tones in the LHM sequences. In the repetition sequences, the final tone is the same as either the first or the second tone; the potential search space, therefore, is smaller and more reliably defined. The third experiment in Endress et al.'s (2007) article addressed this unpredictability in ordinal sequences by maintaining the interval between the first and third tones as a constant two tones, and this improved learning.

We adapted the $\mathrm{ABA} / \mathrm{ABB}$ sequences from Endress et al. (2007) to test whether identical repetition promoted learning more than did conditions in which the final tone varied from the first or second tone by either one or two semitones. If similarity contributes in a graded manner to learning, the identical repetition condition should be learned more easily than the one-semitone conditionwhich in turn should be learned more easily than the twosemitone condition. If identical repetition is qualitatively distinct from similarity, only the identical repetition condition should differ from the one- and two-semitone conditions. We assume here that differences of zero, one, and two semitones are judged by participants to be declining in similarity. Harris (1985) directly tested participants' judgments of tone similarity by playing pairs of tones and found that tones distinct by two semitones were judged to be less similar than one-semitone-distinct tones.

\section{Method}

Participants. Nine male and 21 female students at the University of York participated in the experiment for course credit or for $£ 2$ each.

Materials. Twenty piano monotones in semitone intervals were generated using a synthesizer (Nyquist, 2007). Semitones ranged from $\mathrm{G}(97 \mathrm{~Hz})$ to a second E-flat $(313 \mathrm{~Hz})$. Each semitone was sampled at $22050 \mathrm{~Hz}$ with 16-bit resolution, and lasted for $400 \mathrm{msec}$.

There were three conditions: "identical repetition," "one-different," and "two-different" (see Figure 1). For ABA and ABB patterns in the identical repetition condition, the second tone was either higher or lower than the first by a minimum of six semitones, and the third tone was identical to the first tone in the ABA pattern, and identical to the second tone in the $\mathrm{ABB}$ condition. In the one-different condition, the third tone was one semitone higher or lower than the first tone in the ABB condition, and $\mathrm{ABB}$ patterns were the same, except third tones were one semitone higher or lower than the second tone. Tones in the two-different condition were two semitones higher or lower than the other tone.

Procedure. Each trial consisted of five piano tone triplets, played through closed cup headphones. The number of each triplet presented was shown on the screen $(1,2,3,4$,) and when "?" appeared, participants were required to decide whether the last triplet conformed to the others (by pressing " $y$ " on a keyboard) or was a different pattern (by pressing " $n$ "). Congruent and incongruent trials were presented randomly, and feedback (correct/incorrect) was presented on the screen after each trial. There were 160 trials in total, of which half were congruent, and the experiment lasted roughly $30 \mathrm{~min}$.

\section{Results and Discussion}

The 160 responses were broken down into eight blocks of 20 responses each. Accuracy for each block was entered as the dependent variable in an ANOVA with time (blocks one to eight), experimental condition (identity, one-different, two-different), and sequence type (ABA, $\mathrm{ABB}$ ) as factors.

There was a main effect of time $[F(7,189)=2.50, p<$ $\left..05, \eta^{2}=.09\right]$, with accuracy increasing with time. There was a significant main effect of condition $[F(1,27)=50.18$, $p<.001, \eta^{2}=.79$ ], with learning significantly higher in the identical repetition condition than in the one-different and two-different conditions (Tukey's test, $p<.001$ ). The one-different and two-different conditions were not significantly different $(p=.68)$. The interaction between time and condition was also significant $[F(14,189)=$ $\left.2.61, p<.005, \eta^{2}=.16\right]$, indicating that there was greater improvement in the identical repetition condition than in the other conditions. No other main effects or interactions were significant (all $F \mathrm{~s}<1$ ). Comparisons with chancelevel performance are shown at each time step in Figure 2. Over all time steps, the identical repetition condition and the two-different condition resulted in significantly better learning than chance $(p<.01)$, but the one-different condition was only marginally better than chance after Bonferroni correction ( $p=.07)$. A summary of the main effect of condition is shown in Figure 5A. Harris (1985) found that the difference in similarity judgments between one- and two-semitone-different pairs of tones was smaller than that between zero- and one-semitone-different pairs, and so the sequence learning results match the psychoacoustic properties of tone similarity.

The results supported the claim, made in Endress et al. (2007), that identical repetition is a special case for learning. Whether the third tone differed by one or two semitones from the first or the second tone had a significant and equal impact on learning compared with when there was no difference in pitch for the third tone. However, the one- and two-different conditions differed from the identical repetition condition in terms of whether the third tone 


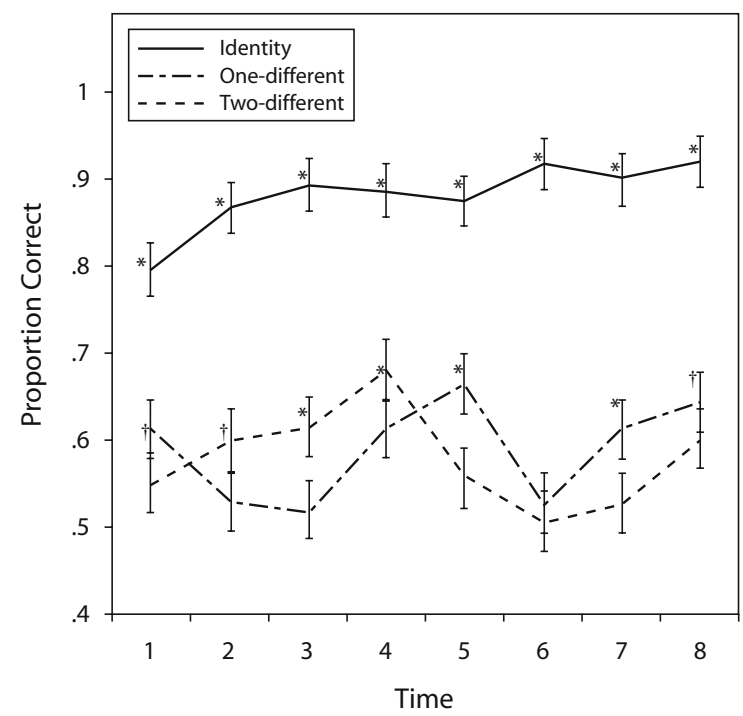

Figure 2. Means for Experiment 1, with variability for one- and two-semitone-different conditions. Indices next to data points indicate difference from chance level. ${ }^{*} p<.05 . \quad{ }^{\dagger} p<.1$.

was precisely predictable from the first two tones or not, since the tone could either rise or fall in pitch, so Experiment 1 tested the combined effects of increasing similarity and variability. Experiment 2 tested whether this effect of variability was critical for the results by repeating the study with the one- and two-different conditions always producing a rise in the third tone, instead of the tone either rising or falling.

\section{EXPERIMENT 2}

\section{Learning From Graded Repetitions Without Variability}

\section{Method}

Participants. Ten male and 20 female students from the University of York participated for course credit or a sum of $£ 2$. None had participated in Experiment 1.

Materials. The materials were identical to those of Experiment 1, except that the third tone in the one-different condition always rose by one semitone over the first tone for ABA sequences or over the second tone for $\mathrm{ABB}$ sequences. The two-different condition always rose by two semitones over the first tone for $\mathrm{ABA}$ sequences or over the second tone for $\mathrm{ABB}$ sequences.

Procedure. The procedure was identical to that of Experiment 1.

\section{Results and Discussion}

As in Experiment 1, we blocked the trials into groups of 20. Time, condition, and sequence type were entered as factors in an ANOVA, with accuracy as a dependent variable. The main effects were qualitatively similar to those for Experiment 1 for time $\left[F(7,189)=2.90, p<.01, \eta^{2}=.10\right]$ and condition $\left[F(2,27)=12.57, p<.001, \eta^{2}=.48\right]$. Identical repetition resulted in significantly higher accuracy than was the case in the other conditions (both $p \mathrm{~s}<.001$ ), but the one- and two-different conditions did not differ $(p=$ .99). As in Experiment 1, the identical repetition condition was qualitatively distinct from the one- and two-different conditions, which resulted in a similar level of learning.

Distinct from Experiment 1, however, was a significant main effect of sequence type $[F(1,27)=11.69, p<.005$, $\left.\eta^{2}=.30\right]$ and a significant interaction between condition and sequence type $\left[F(2,27)=4.56, p<.05, \eta^{2}=.25\right]$. This was due to no significant difference between ABA and $\mathrm{ABB}$ sequences for the identical repetition and twodifferent conditions (both $p s>.2$ ) but a significant advantage for $\mathrm{ABB}$ over $\mathrm{ABA}$ sequences for the one-different condition (mean correct $=.63$ and .73 , respectively) $(p<$ $.05)$; see Figure 3. Predictable differences in pitch between very similar but not identical tones resulted in a slightly different strategy for learning the sequences, where perhaps the final bigram of each tone triple determined per-

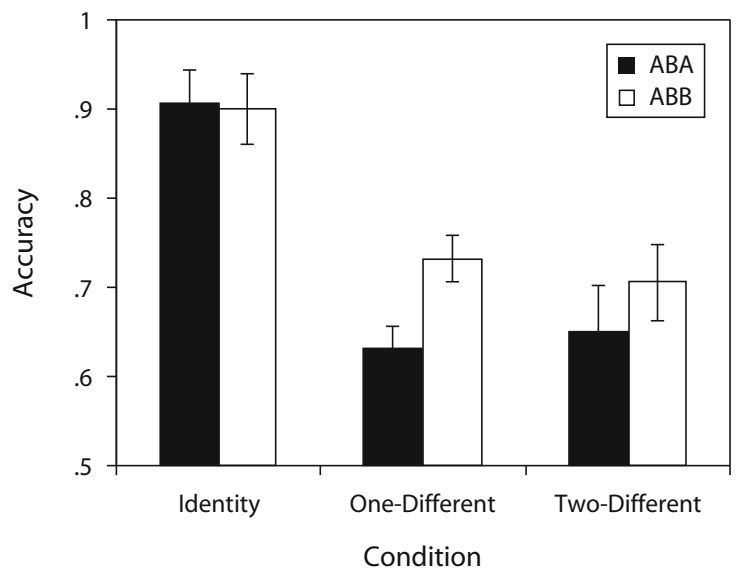

Figure 3. Effect of condition on $\mathrm{ABA}$ and $\mathrm{ABB}$ sequences in Experiment 2.

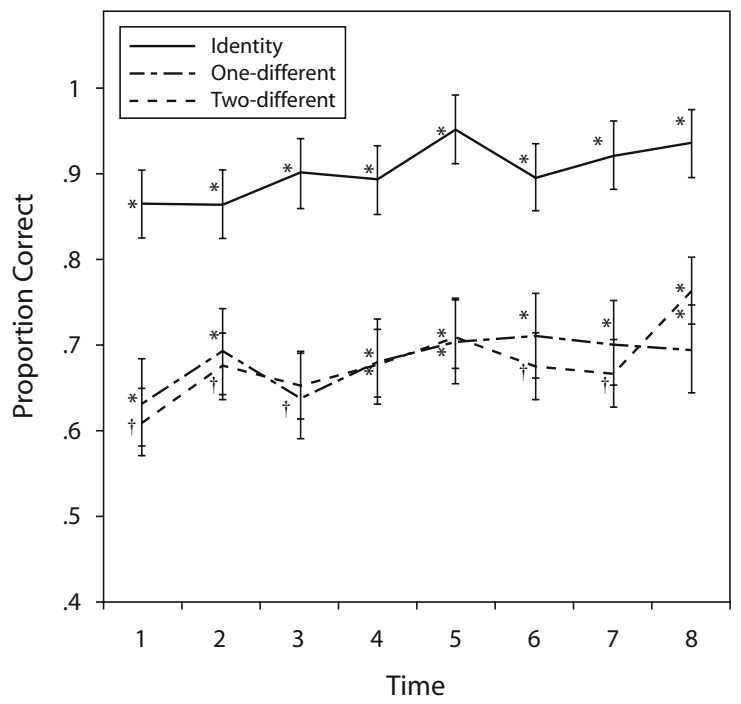

Figure 4. Means for Experiment 2, with no variability for oneand two-semitone-different conditions. ${ }^{*} p<.05$. ${ }^{\dagger} p<.1$. 
A

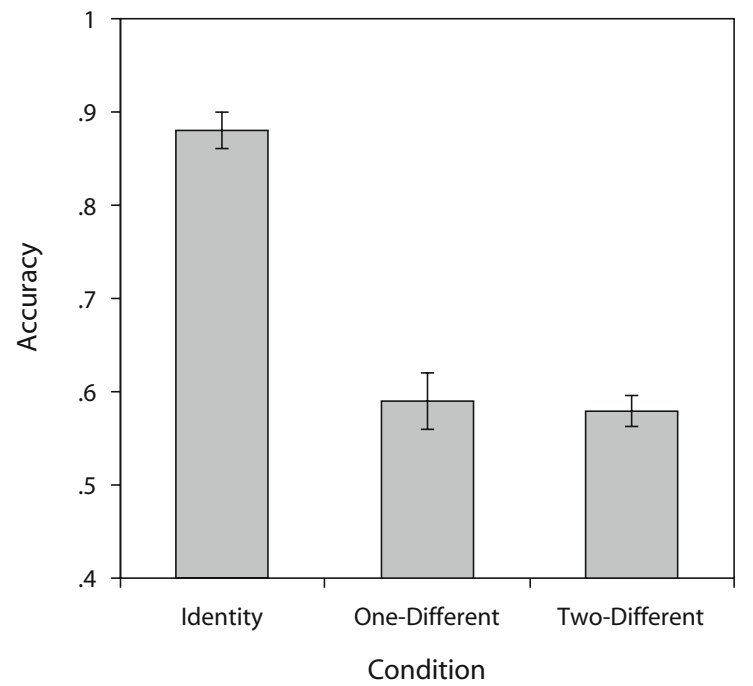

B

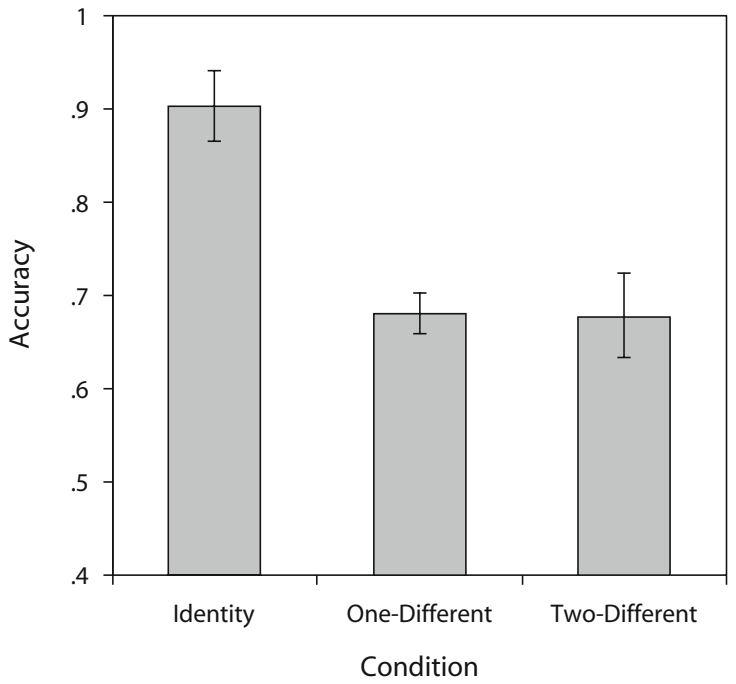

Figure 5. Summary of results from (A) Experiment 1 and (B) Experiment 2, showing means and standard errors of the means across the three conditions.

formance: Participants listened for whether the final two tones rose by a single semitone, or whether they described a larger interval. The other interactions were not significant (all $F_{\mathrm{S}} \leq 1.09$ ). Comparisons to chance level for each time block are shown in Figure 4. Over all blocks, all three conditions were significantly better than chance (all $p \mathrm{~s}<$ .01 ), indicating that predictability resulted in less variable responses in the one- and two-different conditions than were obtained in Experiment 1. Figure 5B shows a summary of the main effect of condition.

\section{GENERAL DISCUSSION}

The results of the two experiments met the first aim of the study, confirming that repetition has a special status in sequence learning. When tones were identical between first and third or second and third positions, learning was significantly better than when the relationship between first and third or second and third tones differed by one or two semitones. The second aim of the study was to determine whether precise repetition figured along a continuum of similarity, or whether it was qualitatively distinct from one- or two-semitone-different sequences. We found that deviating from identical repetition by either a semitone or a tone had an equal and catastrophic impact on learning, indicating that, at least for tone sequences, there was a step change in learning from repeated rather than unrepeated but similar sequences. This close experimental comparison between conditions of repetition and nonidentical similarity goes beyond previous demonstrations of the importance of similarity for scaffolding learning of word or grammatical structure (Bonatti et al., 2005; Newport \& Aslin, 2004; Onnis et al., 2005; Pothos \& Bailey, 2000 ), in that we have shown that precise repetitions provide the strongest constraints on sequence learning.
The third aim was to determine the relative effect of variability on learning sequences with different degrees of similarity between tones. We found that variability did not have a profound quantitative impact on learning over and above the extent of similarity. For both Experiment 1 (with variability) and Experiment 2 (without) we found similar levels of performance for the one- and two-semitone-different conditions. An ANOVA with experiment, condition, time, and sequence type revealed no significant main effect or interactions of experiment (all $F \mathrm{~s}<1)$. However, there was indirect evidence that no variability resulted in better learning over time of the sequence structure - the significant interaction between time and condition from Experiment 1 (where the identical repetition resulted in faster learning) was not replicated in Experiment 2, because of some evidence of learning in each of the conditions in this study (compare Figures 2 and 4). Furthermore, the learning of $\mathrm{ABA}$ and $\mathrm{ABB}$ sequences appeared to be slightly different in the two experiments. In Experiment 1, there was no advantage of one sequence type over the other, but in Experiment 2 , for the one-different condition, $\mathrm{ABB}$ sequences were learned more easily than $\mathrm{ABA}$ sequences were. This indicates that participants' performance may be somewhat strategic for these tasks; altering one characteristic of the stimuli, from variable to nonvariable, resulted in different response to the stimuli. The better learning of ABB sequences, consistent with Endress et al.'s (2007) studies, was only reliably found for the one-semitone-different condition, perhaps indicating sensitivity to bigrams for this contrast: Participants preferentially respond to a rise by one semitone (in the $\mathrm{ABB}$ sequences), compared with a change greater than two semitones (as in the ABA sequence). However, although participants may be learning from bigrams in this condition, note that bigrams are an equally effective strategy in all conditions in the experiments. Thus, the com- 
parison between zero-, one-, and two-semitone-different sequences can still be made directly, even if it is unclear whether participants are learning only from adjacent tones, or across nonadjacencies as well.

Whatever the locus of the effect of repetition in the statistical structure of the sequences, our studies have confirmed that repetition can guide learning, and that it is a special case as a constraint for detecting structures. Such effects are consistent with the effects of repetition in perceptual and memory tasks (e.g., Henson, 1998; Kanwisher, 1987); consequently, repetition in speech to children is likely to have a profound effect in learning word boundaries and grammatical structure (e.g., Onnis et al., 2005). What sort of learning mechanism can account for these effects? An adequate learning system must be able to account for (1) sensitivity to repetition that is discontinuous with respect to similarity, and (2) learning of the structure that is guided by repetition. The present data do not constrain the architecture of the cognitive system for the latter point; repetition, once detected, may then pass onto either an associative learning mechanism (Altmann, 2002) or a symbolic processing system (Marcus et al., 1999).

So the point at issue is the implementation of repetition sensitivity in the cognitive system. Endress et al. (2007) claimed that the special status of repetition requires a modular perceptual mechanism that detects identity in sequences. But is repetition sensitivity also compatible with general-purpose learning mechanisms? The effects of similarity in sequences are likely to be available to computational models that respond to the statistical structure of the input. Indeed, detecting similarity (and representing such similarity within the model) is a fundamental principle in self-organizing neural network models of cognition (e.g., Hopfield, 1982; Kohonen, 1988); yet such models would predict that repetition is on the same continuum as stimuli that are not identical but are perceptually similar, which was not the case in the present experiments. However, statistically based model learning sequences that contain repetitions would be required to distinguish different occurrences of the repeating stimuli at different points in the sequence; such a mechanism could be generated without requiring a perceptual mechanism. One possible modeling framework, for instance, is the recurrent backpropagation model of short-term memory by Botvinick and Plaut (2006), where the representation of stimuli in the hidden units of the model is a function of the stimulus identity and its sequence position. Whether such a model would reflect the qualitative distinction between identical repetition and similarity is an open question, but the respecting of both featural and temporal similarity in this model provides some promise for simulations of the human data without requiring a specific cognitive architecture for processing repetitions.

\section{AUTHOR NOTE}

Correspondence concerning this article should be addressed to P. Monaghan, Department of Psychology, Lancaster University, Lancaster LA1 4YF, England (e-mail: p.monaghan@lancaster.ac.uk).

\section{REFERENCES}

Altmann, G. T. M. (2002). Learning and development in neural networks - the importance of prior experience. Cognition, 85, B43B50.

Bonatti, L. L., Peña, M., Nespor, M., \& Mehler, J. (2005). Linguistic constraints on statistical computations: The role of consonants and vowels in continuous speech processing. Psychological Science, 16, 451-459.

Botvinick, M., \& Plaut, D. C. (2006). Short-term memory for serial order: A recurrent neural network model. Psychological Review, 113, 201-233.

Endress, A. D., Dehaene-Lambertz, G., \& Mehler, J. (2007). Perceptual constraints and the learnability of simple grammars. Cognition, 105, 577-614.

Gómez, R. (2002). Variability and detection of invariant structure. Psychological Science, 13, 431-436.

HARRIs, R. W. (1985). Perceived relatedness of musical tones in major and minor tonal contexts. American Journal of Psychology, 98, 605-623.

Henson, R. N. A. (1998). Item repetition in short-term memory: Ranschburg repeated. Journal of Experimental Psychology: Learning, Memory, \& Cognition, 24, 1162-1181.

Hopfield, J. J. (1982). Neural networks and physical systems with emergent collective computational abilities. Proceedings of the $\mathrm{Na}$ tional Academy of Sciences, 79, 2554-2558.

KANwISHER, N. G. (1987). Repetition blindness: Type recognition without token individuation. Cognition, 27, 117-143.

KoHonen, T. (1988). The "neural" phonetic typewriter. Computer, 21, 11-22.

Marcus, G. F., ViJayan, S., Rao, S. B., \& Vishton, P. M. (1999). Rule learning by seven-month-old infants. Science, 283, 77-80.

NewPort, E. L., \& AsLin, R. N. (2004). Learning at a distance I. Statistical learning of non-adjacent dependencies. Cognitive Psychology, 48, 127-162.

NYQUIST (www.cs.cmu.edu/ music/nyquist/). Retrieved May 6, 2007.

Onnis, L., Christiansen, M. H., Chater, N., \& Gómez, R. (2003). Reduction of uncertainty in human sequential learning: Evidence from artificial grammar learning. Proceedings of the 25th Annual Conference of the Cognitive Science Society. Mahwah, NJ: Erlbaum.

Onnis, L., Monaghan, P., Richmond, K., \& Chater, N. (2005). Phonology impacts segmentation in online speech processing. Journal of Memory \& Language, 53, 225-237.

Papousek, M., Papousek, H., \& Haekel, M. (1987). Didactic adjustments in fathers' and mothers' speech to their 3-month-old infants. Journal of Psycholinguistic Research, 16, 491-516.

Peña, M., Bonatti, L. L., Nespor, M., \& Mehler, J. (2002). Signaldriven computations in speech processing. Science, 298, 604-607.

Pothos, E. M., \& Bailey, T. M. (2000). The importance of similarity in artificial grammar learning. Journal of Experimental Psychology: Learning, Memory, \& Cognition, 26, 847-862.

SAFFran, J. R. (2003). Statistical language learning: Mechanisms and constraints. Current Directions in Psychological Science, 12, 110-114.

(Manuscript received March 3, 2008; revision accepted for publication August 23, 2008.) 